\title{
Influence of the Mutation Operator on the Solution of an Inverse Stefan Problem by Genetic Algorithms
}

\author{
Damian Słota \\ Institute of Mathematics, Silesian University of Technology, \\ Kaszubska 23, 44-100 Gliwice, Poland
}

\begin{abstract}
This paper presents the influence of choice of the mutation operator on the accuracy of a solution of a two-phase design inverse Stefan problem using genetic algorithms. In the problem to be solved, the coefficient of convective heat transfer on one boundary had to be so selected that the moving interface of the phase change (freezing front) would take the given position.
\end{abstract}

\section{Introduction}

In this paper we are going to find a solution of a two-phase design inverse Stefan problem [1], for which the coefficient of convective heat transfer on one boundary should be so selected that the moving interface could take the given position. The solution will consist in minimization of the functional whose value is the norm of the difference between the given interface position and the position reconstructed for the selected convective heat-transfer coefficient. For the minimization of the functional genetic algorithms were used, whereas the Stefan problem was solved by an alternating phase truncation method 4 . The paper presents the influence of choice of the mutation operator on the accuracy of the results obtained.

\section{Formulation of the Problem}

On the boundary of domain $D=[0, b] \times\left[0, t^{*}\right] \subset \mathbb{R}^{2}$ three components are distributed $\Gamma_{0}=\{(x, 0) ; x \in[0, b]\}, \Gamma_{1}=\left\{(0, t) ; t \in\left[0, t^{*}\right]\right\}, \Gamma_{2}=\left\{(b, t) ; t \in\left[0, t^{*}\right]\right\}$, where initial and boundary conditions are given. Let $D_{1}\left(D_{2}\right)$ be this subset of domain $D$ which is occupied by liquid (solid) phase, separated by the freezing front $\Gamma_{g}=\xi(t)$.

We will look for an approximate solution of the following problem. For given position of freezing front $\Gamma_{g}$, the distribution of temperature $T_{k}$ in domain $D_{k}$ $(k=1,2)$ is calculated as well as function $\alpha(t)$ on boundary $\Gamma_{2}$, which satisfy the following equations (for $k=1,2$ ):

$$
\begin{aligned}
\frac{\partial T_{k}}{\partial t}(x, t) & =a_{k} \frac{\partial^{2} T_{k}}{\partial x^{2}}(x, t), & & \text { in } D_{k}, \\
T_{1}(x, 0) & =\varphi_{0}(x), & & \text { on } \Gamma_{0},
\end{aligned}
$$




$$
\begin{aligned}
\frac{\partial T_{k}}{\partial x}(x, t) & =0, & & \text { on } \Gamma_{1}, \\
-\lambda_{k} \frac{\partial T_{k}}{\partial x}(x, t) & =\alpha(t)\left(T_{k}(x, t)-T_{\infty}\right), & & \text { on } \Gamma_{2}, \\
T_{k}(x, t) & =T^{*}, & & \text { on } \Gamma_{g}, \\
L \varrho_{2} \frac{d \xi}{d t} & =-\lambda_{1} \frac{\partial T_{1}(x, t)}{\partial x}+\lambda_{2} \frac{\partial T_{2}(x, t)}{\partial x}, & & \text { on } \Gamma_{g},
\end{aligned}
$$

where $a_{k}$ are the thermal diffusivity in liquid phase $(k=1)$ and solid phase $(k=2), \lambda_{k}$ are the thermal conductivity, $\alpha$ is the coefficient of convective heattransfer, $T_{\infty}$ is the ambient temperature, $L$ is the latent heat of fusion, $\varrho_{k}$ are the mass density, and $t$ and $x$ refer to time and spatial location, respectively.

We will look for the $\alpha(t)$ function in the form:

$$
\alpha(t)= \begin{cases}\alpha_{1} & \text { for } t \leq t_{\alpha_{1}}, \\ \alpha_{2} & \text { for } t \in\left(t_{\alpha_{1}}, t_{\alpha_{2}}\right], \\ \alpha_{3} & \text { for } t>t_{\alpha_{2}},\end{cases}
$$

where $0<t_{\alpha_{1}}<t_{\alpha_{2}}<t^{*}$. Let $V_{\alpha}^{p}$ mans a set of all functions in the form (77), where $\alpha_{i} \in\left[\alpha_{i}^{l}, \alpha_{i}^{u}\right]$. For the given function $\alpha(t) \in V_{\alpha}^{p}$ the problem (11)-(6) becomes a direct Stefan problem, whose solution enables finding the position of the interface $\xi(t)$ corresponding to the $\alpha(t)$ function. Using the found interface position $\xi(t)$ and the given position $\xi^{*}(t)$ we can build a functional which will specify the error of an approximated solution:

$$
J(\alpha)=\left(\sum_{i=1}^{M}\left[\omega_{i}\left(\xi_{i}-\xi_{i}^{*}\right)^{2}\right]\right)^{1 / 2},
$$

where $\omega_{i}$ are weight coefficients and $\xi_{i}^{*}=\xi^{*}\left(t_{i}\right)$ and $\xi_{i}=\xi\left(t_{i}\right)$ are the given and calculated points, respectively, describing the moving interface position.

\section{Genetic Algorithm}

For the representation of the vector of decision variables $\left(\alpha_{1}, \alpha_{2}, \alpha_{3}\right)$, a chromosome was used in the form of a vector of three real numbers (real number representation) [2,3]. The tournament selection and elitist model were applied in the algorithm. As the crossover operator, arithmetic crossover was applied.

The results of calculations were then compared for different mutation operators: uniform mutation (M1), Gaussian mutation (M2) and two operators of nonuniform mutation ( $M 3$ and $M 4$ ) for different functions describing the uniformity of distribution. In the case of uniform mutation (M1) the $\alpha_{i}$ gene is transformed according to the equation:

$$
\alpha_{i}^{\prime}=\alpha_{i}^{l}+r\left(\alpha_{i}^{u}-\alpha_{i}^{l}\right),
$$

where $r$ is a random number with a uniform distribution from the domain $[0,1]$, and $\alpha_{i}^{u}$ and $\alpha_{i}^{l}$ are the upper and lower limits, respectively, of variability interval 
of the $\alpha_{i}$ parameter, i.e. $\alpha_{i} \in\left[\alpha_{i}^{l}, \alpha_{i}^{u}\right]$. In the case of Gaussian mutation (M2) the $\alpha_{i}$ gene is transformed according to the equation:

$$
\alpha_{i}^{\prime}=\alpha_{i}+r(\tau)
$$

where $r(\tau)$ is a random number with normal distribution with mean value equal to zero and variance equal to:

$$
\sigma^{2}(\tau)=\frac{N-\tau}{N} \frac{\left(\alpha_{i}^{u}-\alpha_{i}^{l}\right)}{3},
$$

where $\tau$ is the current generation number, $N$ is the maximum number of generations. In the calculations, a nonuniform mutation operator was used as well. During mutation, the $\alpha_{i}$ gene is transformed according to the equation:

$$
\alpha_{i}^{\prime}=\left\{\begin{array}{l}
\alpha_{i}+\Delta\left(\tau, \alpha_{i}^{u}-\alpha_{i}\right), \\
\alpha_{i}-\Delta\left(\tau, \alpha_{i}-\alpha_{i}^{l}\right),
\end{array}\right.
$$

and a decision is taken at random which from the above formulas should be applied. Function $\Delta(\tau, x)$ was assumed in the form (M3 and $M 4$, respectively):

$$
\Delta_{3}(\tau, x)=x\left(1-r^{\left(1-\frac{\tau}{N}\right) d}\right) \quad \text { or } \quad \Delta_{4}(\tau, x)=x r\left(1-\frac{\tau}{N}\right)^{d},
$$

where $r$ is a random number with a uniform distribution from the domain $[0,1]$, $\tau$ is the current generation number, $N$ is the maximum number of generations and $d$ is a constant parameter (in the calculations, $d=2$ was assumed).

\section{Calculations}

It was assumed in the calculations that: $b=0.08, a_{k}=\lambda_{k} /\left(c_{k} \varrho_{k}\right)$ for $k=1,2$, $\lambda_{1}=33, \lambda_{2}=30, c_{1}=800, c_{2}=690, \varrho_{1}=7000, \varrho_{2}=7500, L=270000$. The temperature of solidification is $T^{*}=1500$, ambient temperature is $T_{\infty}=50$ and initial temperature is equal $\varphi_{0}(x)=1540$. The exact value of the convective heat transfer coefficient amounts to:

$$
\alpha(t)= \begin{cases}1200 & \text { dla } t \leq 38, \\ 800 & \text { dla } t \in(38,93], \\ 250 & \text { dla } t>93 .\end{cases}
$$

For each of the mutation operators and different probability values of crossover $\left(p_{c}\right)$ and mutation $\left(p_{m}\right)$, calculations were carried out for ten different initial settings of a pseudorandom numbers' generator.

In the case of Gaussian mutation (M2), the best results were obtained for the crossover probability $p_{c}=0.75$ and for mutation probability $p_{m}=0.01$; the average value of the minimum found was 0.00245326 , and the average value of the minimum point found $\alpha_{a v g}=(1203.179,792.496,250.506)$. 
For the remaining mutation operators, a zero value of the minimized functional was obtained. In the case of a uniform mutation (M1) and a nonuniform mutation with $\Delta_{3}(\tau, x)$ function (M3), the zero value was obtained twice, for $p_{c} \in$ $\{0.7,0.75\}$ and $p_{m}=0.1$. In the case of a nonuniform mutation with $\Delta_{4}(\tau, x)$ function $\left(M_{4}\right)$, this value was obtained three times, for $p_{c} \in\{0.7,0.75,0.8\}$ and $p_{m}=0.1$. The results with the same value of the objective function can be subjected to further evaluation due to errors in the convective heat-transfer coefficient reconstruction. The least errors were obtained for a nonuniform mutation with $\Delta_{3}(\tau, x)$ function (M3) and $p_{m}=0.1$ and $p_{c}=0.7$. The values found for the reconstructed coefficient are $\alpha_{\text {avg }}=(1200.003,800.008,249.999)$. A not much worse result was obtained for the same operator and crossover probability equal 0.75. In that case, the values founds were $\alpha_{a v g}=(1200.01,800.006,249.99)$. In the remaining cases, the convective heat-transfer coefficient values were reconstructed with greater errors. Thus, in the case of the nonuniform mutation with $\Delta_{4}(\tau, x)$ function $\left(M_{4}\right)$, the following values were found: $\alpha_{a v g}=$ $(1199.934,800.072,249.996)$ for $p_{c}=0.8, \alpha_{a v g}=(1200.037,799.991,249.998)$ for $p_{c}=0.75, \alpha_{a v g}=(1200.190,799.863,250.003)$ for $p_{c}=0.7$. For the uniform mutation $(M 1)$, the values determined were as follows: $\alpha_{a v g}=(1200.197,799.861$, $250.001)$ for $p_{c}=0.75, \alpha_{a v g}=(1200.344,799.720,250.012)$ for $p_{c}=0.7$.

Calculations for other values of the genetic algorithm parameters were also made, however, for none of the sets of values better results were obtained than those presented in this paper.

\section{Conclusion}

The paper presents the influence of choice of the mutation operator on the accuracy of a solution to the two-phase design inverse Stefan problem using genetic algorithms. The problem under consideration consisted in such selection of a convective heat transfer coefficient on one boundary that the moving interface would take the given position. Results for a uniform mutation, nonuniform mutation and Gaussian mutation have been presented. The best results were obtained for the nonuniform mutation with $\Delta_{3}(\tau, x)$ function and mutation probability $\left(p_{m}\right)$ equal 0.1 and crossover probability $\left(p_{c}\right)$ equal 0.7 . The calculation results obtained show a very good approximation of the exact solution, thus corroborating the usefulness of the presented approach.

\section{References}

1. Goldman, N.L.: Inverse Stefan Problem. Kluwer, Dordrecht (1997)

2. Michalewicz, Z.: Genetic Algorithms + Data Structures = Evolution Programs. Springer-Verlag, Berlin (1996)

3. Osyczka, A.: Evolutionary Algorithms for Single and Multicriteria Design Optimization. Physica-Verlag, Heidelberg (2002)

4. Rogers, J.C.W., Berger, A.E., Ciment, M.: The Alternating Phase Truncation Method for Numerical Solution of a Stefan Problem. SIAM J. Numer. Anal. 16 (1979) 563-587 